\title{
ÜBERSICHT
}

\section{Zu den Zusammenhängen zwischen paraphilen Störungen, Persönlichkeitsstörungen und Sexualdelinquenz}

\author{
Theres Anna Hörburger ${ }^{1,2}$ • Elmar Habermeyer ${ }^{3}$ \\ Eingegangen: 2. Januar 2020 / Angenommen: 6. März 2020 / Online publiziert: 31. März 2020 \\ (c) Der/die Autor(en) 2020
}

\section{Zusammenfassung}

Studien zeigen einen robusten Zusammenhang zwischen psychischen Störungen und Sexualdelinquenz. Im Vergleich zur Allgemeinbevölkerung sind bei Sexualstraftätern sowohl paraphile Störungen als auch Persönlichkeitsstörungen überrepräsentiert. Auch zeigen persönlichkeits- und paraphil gestörte Sexualstraftäter gegenüber Sexualdelinquenten ohne solche Diagnosen neben anderen psychischen Störungen häufiger psychosoziale Auffälligkeiten, Schwierigkeiten im Bereich der Behandlung und ungünstigere Legalprognosen. Es lassen sich zudem deliktspezifische Unterschiede beobachten: Vergewaltiger und Sexualmörder weisen diagnostisch eher Persönlichkeitsstörungen aus dem Cluster B und einen sexuellen Sadismus auf, während Täter mit Kindern als Opfern vermehrt im Cluster C zu finden sind und mit einer Pädophilie diagnostiziert werden. Diskutiert werden unterschiedliche Hypothesen zu den Verbindungen zwischen Sexualdelinquenz und Persönlichkeits- sowie paraphilen Störungen. Diagnostisch relevante Normabweichungen in Sexualität und Persönlichkeit bieten zwar keine monokausale Erklärung für die Entstehung und Aufrechterhaltung von Sexualdelinquenz, sind jedoch oftmals elementar für ein umfassendes Deliktverständnis und bedürfen in der Planung und Umsetzung der Behandlung von Sexualstraftätern besonderer Aufmerksamkeit.

Schlüsselwörter Sexualdelikt · Paraphilie · Persönlichkeit · Delinquenz · Psychische Störung

Die vorliegende Arbeit wurde verfasst im Rahmen des

Weiterbildungsmasters Psychologie mit Schwerpunkt Forensische Psychologie an der Universität Konstanz.

Theres Anna Hörburger, M.Sc. Psychologie

theres.hoerburger@uni-konstanz.de,

theres.hoerburger@ji.zh.ch
1 Psychiatrisch-Psychologischer Dienst, Justizvollzug und Wiedereingliederung Zürich, Hohlstrasse 552, 8090 Zürich, Schweiz

2 Universität Konstanz, Konstanz, Deutschland

3 Klinik für Forensische Psychiatrie, Psychiatrische Universitätsklinik Zürich, Zürich, Schweiz 


\title{
Connections between paraphilic disorders, personality disorders and sexual delinquency
}

\begin{abstract}
Studies have shown a robust relationship between mental disorders and sexual delinquency. Compared to the general population both paraphilic disorders and personality disorders are overrepresented in sex offenders. Furthermore, sex offenders with personality disorders and paraphilic disorders more often show psychiatric comorbidities, psychosocial abnormalities, difficulties in the area of treatment and less favorable legal prognoses compared to sex offenders without such diagnoses. In addition, offence-specific differences can be observed: rapists and sexual murderers are more likely to have personality disorders from cluster B and sexual sadism, while offenders with children as victims are more likely to be diagnosed with cluster $\mathrm{C}$ personality disorders and pedophilia. Different hypotheses on the connections between sexual delinquency, personality disorders and paraphilic disorders are discussed. Although diagnostically relevant, normal deviations in sexuality and personality do not provide a monocausal explanation for the development and maintenance of sexual delinquency; however, they are often crucial for a comprehensive approach and require special attention in the planning and implementation of the treatment for sexual offenders.
\end{abstract}

Keywords Sex crime $\cdot$ Paraphilia $\cdot$ Personality $\cdot$ Delinquency $\cdot$ Mental illness

\section{Psychische Störungen bei (Sexual-)Delinquenten}

Die im Vergleich zur Allgemeinbevölkerung erhöhte Prävalenz psychischer Störungen bei Straftätern ist in der Literatur gut dokumentiert (Laajasalo et al. 2013; Fazel et al. 2007; Långström et al. 2004). Während in der Allgemeinbevölkerung zwischen 20 und $30 \%$ der Männer unter einer psychischen Störung leiden (Steel et al. 2014), sind in Gefängnispopulationen 48-73\% der männlichen Insassen betroffen (Fazel und Danesh 2002). Mehr als die Hälfte der Gefängnisinsassen erfüllen die diagnostischen Kriterien für mehr als eine psychische Störung (Al-Rousan et al. 2017).

Die Gruppe der Sexualstraftäter scheint nochmals häufiger psychische Störungen aufzuweisen, denn in einer repräsentativen österreichischen Studie wurden bei $92 \%$ der über 1000 untersuchten Sexualstraftäter mindestens eine, bei $80 \%$ sogar 2 oder mehr psychiatrische Diagnosen gestellt (Eher et al. 2019). Die häufigsten Diagnosen bei Sexualstraftätern sind Persönlichkeits-, Substanzgebrauchsund paraphile Störungen (Hoyer et al. 1999; Chan und Heide 2009; Briken et al. 2010; Chan et al. 2015; Khachatryan et al. 2016; Koch et al. 2011; Myers et al. 2016, 2010). Nachfolgend wird eine Übersicht über die bisherigen empirischen Erkenntnisse zu den Zusammenhängen zwischen Sexualdelinquenz und paraphilen Störungen bzw. Persönlichkeitsstörungen sowie deren Kombination gegeben. Die Berücksichtigung dieser Zusammenhänge im Rahmen eines individuellen Behandlungskonzepts kann Rückfallrisiken bei Sexualstraftätern effektiv senken (Helmus et al. 2009).

\section{Paraphilie und paraphile Störungen}

Diagnostisch bzw. klinisch relevante sexuelle Normabweichungen werden in der ICD-10 als „Störungen der Sexualpräferenz" zusammengefasst (Dilling und Freyberger 2012). Hierbei wird nicht unterschieden zwischen sexuellen Präferenzen, die ungewöhnlich sind bzw. wegen ihrer Abweichung von normativen Wertvorstellungen erfasst werden, und Störungen, die mit einem Leidensdruck bzw. nachteiligen Folgen für andere Personen verbunden sind. Vielmehr erfordert die ICD-10-Diagnose einer Störung der Sexualpräferenz beides, obwohl der Aspekt der Normabweichung stark mit dem aktuellen gesellschaftspolitischen Klima zusammenhängt und einem beständigen Wandel unterliegt, wie sich beispielsweise anhand der Homosexualitäts-Debatte der 1990er zeigte (Fiedler 2004).

Diesem Umstand trägt das DSM-5 (APA 2014) Rechnung, indem es zwischen „Paraphilien“ und „paraphilen Störungen“ differenziert. Unter „Paraphilien“ (von griechisch pará, ,,abseits“, ,,neben“, und philía, „Freundschaft“, „Liebe“) werden hierbei alle intensiven, anhaltenden sexuellen Neigungen verstanden, die sich nicht auf sexuelle Handlungen an und mit phänotypisch normalen, körperlich erwachsenen und einwilligenden Menschen beziehen. Eine Paraphilie als solche rechtfertigt und erfordert jedoch noch keine therapeutische Intervention. Infolge dieser definitorischen Kriterien kann die Prävalenz für Paraphilien in der Allgemeinbevölkerung nur schwer ausgemacht werden. Es gibt allerdings viele Hinweise darauf, dass paraphile Interessen in der Allgemeinbevölkerung nicht ungewöhnlich und auch nicht per se mit Sexualdelinquenz verknüpft sind (Watts et al. 2019). Unter männlichen Befragten, die im Schnitt mehr Interesse an paraphilen Aktivitäten zeigen als Frauen, berichten zwischen 64 und $95 \%$ von paraphilen (fetischistischen, sadistischen oder voyeuristischen) Fan- 


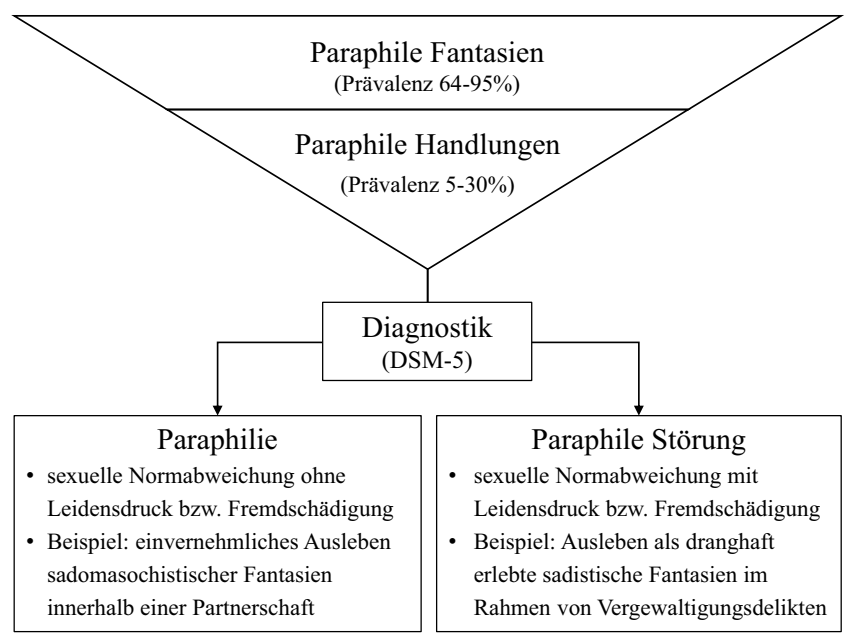

Abb. 1 Paraphile Fantasien, Handlungen und zugehörige Diagnosen

tasien. Diese werden jedoch nur in einer kleinen Zahl der Fälle (zwischen 5 und 30\%) tatsächlich ausgelebt (Williams et al. 2009; Ahlers et al. 2011; Agnew 2001; Dawson et al. 2016).

Entsprechend sind Paraphilien nicht strafrechtlich relevant, denn andere Personen werden aufgrund der zugehörigen Interessen nicht gegen ihren Willen oder im Zustand mangelnder Einwilligungsfähigkeit in sexuelle Handlungen miteinbezogen. Dies trifft erst auf paraphile Störungen zu, denn hier führt die entsprechende Neigung zu signifikantem Leiden oder Beeinträchtigungen der Betroffenen bzw. ist deren Befriedigung mit der Schädigung anderer Personen verbunden (Niemeczek 2015; American Psychiatric Association 2014). Da es in den nächsten Abschnitten um die Zusammenhänge zwischen paraphilen Normabweichungen und Delinquenz geht, die in der Literatur zumeist anhand von Straftäterpopulationen untersucht und diskutiert wurden (Osterheider et al. 2012), wird im Anschluss, der Terminologie des DSM-5 folgend, von paraphilen Störungen gesprochen. Damit sind auch Störungen der Sexualpräferenz nach den Vorgaben der ICD-10 gemeint, die strafrechtlich relevant geworden sind.

Dass es nachfolgend nur um eine kleine, für den Bereich der forensischen Psychiatrie und Psychotherapie allerdings hoch relevante Subgruppe der Menschen mit sexuellen Normabweichungen gehen wird, kann am Beispiel der Pädophilie dargestellt werden: Während pädosexuelle Fantasien von 9,5\% der Männer berichtet werden (Ahlers et al. 2011), liegt die geschätzte Prävalenz pädophiler Störungen in der männlichen Bevölkerung zwischen 3 und $5 \%$ (American Psychiatric Association 2014). In 2 deutschen Untersuchungen an einer Allgemeinbevölkerungsstichprobe lag der Anteil an Hinweisen auf pädophile Störungen mit $0,1 \%$ bzw. $1 \%$ (Dombert et al. 2016; Ahlers et al. 2011) nochmals deutlich niedriger.

Die vorab skizzierten Zusammenhänge illustriert Abb. 1.

\section{Paraphile Störungen und Sexualdelinquenz}

Zwar kann nicht bei allen Sexualstraftätern die Diagnose einer Paraphilie oder paraphilen Störung gestellt werden (Bogaerts et al. 2008), jedoch ist ihr Anteil mit 43-98\% gegenüber der Prävalenz in der Normalbevölkerung deutlich erhöht (Eher et al. 2019; McElroy et al. 1999; Jackson und Richards 2007; Dunsieth et al. 2004). Zudem finden sich bei Sexualstraftätern häufig parallel, d.h. komorbid, mehrere paraphile Störungen (Bradford et al. 1992). In einer US-amerikanischen Stichprobe wiesen $85 \%$ der untersuchten Sexualstraftäter mindestens 2 Paraphiliediagnosen auf (Abel 1985). Bei Sexualstraftätern sind die am häufigsten berichteten paraphilen Störungen Pädophilie und sexueller Sadismus (Berner et al. 2003; Chan und Heide 2009; McElroy et al. 1999; Jackson und Richards 2007; Myers et al. 2010). Dabei erfüllt zwischen einem Drittel und der Hälfte der Kindesmissbrauchstäter die Kriterien für eine pädophile Störung (Eher et al. 2010, 2019; Fromberger et al. 2013).

Zwischen Sexualstraftätern mit und ohne paraphile Störungen konnten deutliche Unterschiede gefunden werden. So weisen Sexualstraftäter mit paraphilen Störungen signifikant mehr psychiatrische Komorbiditäten und eine vielseitigere kriminelle Vorgeschichte auf (Eher et al. 2003). Zudem zeigen sie in einer Stichprobe von Männern mit sexuellen Tötungsdelikten häufiger Entwicklungsprobleme, eine höhere Anzahl vorhergehender Sexualdelikte, mehr Serienmorde, mehr dem Täter unbekannte Opfer, mehr sexuellen Sadismus und mehr zwanghaftes Masturbationsverhalten (Briken et al. 2006). In 2 österreichischen Studien wiesen die Täter mit kindlichen Opfern im Vergleich zu jenen mit erwachsenen Opfern deutlich häufiger paraphile Störungen auf, darunter v. a. Pädophilie und Exhibitionismus. Im Gegensatz dazu zeigten Vergewaltiger häufiger sexuellen Sadismus (Eher et al. 2010, 2019). Täter mit fremden Opfern erfüllten häufiger die Kriterien für Pädophilie, Exhibitionismus, Fetischismus und Voyeurismus. Zehn Prozent dieser Täter erhielten 3 und mehr Paraphiliediagnosen (Eher et al. 2010).

Bei Sexualstraftätern, die ihre Opfer töten, ließen sich mit 52-82\% signifikant häufiger paraphile Störungen diagnostizieren als bei Sexualstraftätern, die dies nicht tun (24-43\%), wobei die häufigsten paraphilen Störungen von Sexualmördern sexueller Sadismus und Pädophilie waren (Briken et al. 2006; Firestone et al. 1998a, 1998b; Koch et al. 2011; Langevin et al. 1988). In einer kanadischen Studie erfüllten Sexualmörder im Vergleich zu Sexualstraftätern, die ihre Opfer nicht töteten, signifikant häufiger die Diagnosekriterien von Exhibitionismus, Fetischismus, Frotteurismus, homosexueller Pädophilie und sexuellem Masochismus (Chan und Beauregard 2016).

Das Vorliegen einer Paraphilie hat sich sowohl bei adoleszenten als auch erwachsenen Sexualdelinquenten als Ri- 
sikofaktor für die Begehung weiterer Sexualstraftaten (Hanson und Morton-Bourgon 2005) erwiesen, nicht jedoch für nichtsexuelle Gewaltstraftaten oder eine allgemeine Rückfälligkeit.

\section{Persönlichkeitsstörungen}

Persönlichkeitsstörungen werden sowohl in der ICD-10 als auch im DSM-5 als stabile, lang andauernde intrapsychische Erlebens- und Verhaltensmuster konzeptualisiert, die deutlich von den Erwartungen der soziokulturellen Umgebung abweichen. Die unflexiblen, tief verwurzelten Muster beginnen in der Adoleszenz oder im frühen Erwachsenenalter und zeigen sich in Abweichungen in Kognition, Affektivität, zwischenmenschlichen Beziehungen und Impulskontrolle. Diese beziehen sich auf vielfältige Bereiche des Verhaltens und verursachen dadurch Leidensdruck bei den Betroffenen oder anderen sowie Beeinträchtigungen in wichtigen sozialen Funktionsbereichen (American Psychiatric Association 2014; Dilling und Freyberger 2012).

Persönlichkeitsstörungen sind in der Allgemeinbevölkerung über alle Cluster hinweg vergleichsweise häufig anzutreffen. Studien gehen von Prävalenzraten zwischen 4,4 und $15,5 \%$ aus, wobei diese für das sonderbar-exzentrische Cluster A (paranoide, schizoide und schizotypische Persönlichkeitsstörung) mit 1,6-6,5\%, für das dramatischemotionale Cluster B (Borderline-, histrionische, antisoziale und narzisstische Persönlichkeitsstörung) mit 1,2-7,0\% und für das ängstlich-vermeidende Cluster $\mathrm{C}$ (vermeidende, dependente, zwanghafte und passiv-aggressive Persönlichkeitsstörung) mit 2,6-10,6\% angegeben werden (Barnow et al. 2010).

\section{Persönlichkeitsstörung und Sexualdelinquenz}

Bei Sexualstraftätern ist die Prävalenz von Persönlichkeitsstörungen mit 33\% (Leygraf 2006) bis 66\% (Berger et al. 1999; Berner et al. 1992) deutlich höher. In verschiedenen Untersuchungen wird von einer großen Spannbreite von Persönlichkeitsstörungen bei Sexualstraftätern berichtet. Während Störungen aus dem Cluster A eher selten eine Rolle spielen, werden besonders häufig Züge aus dem Cluster B (v.a. antisozial und narzisstisch) und C (vorwiegend dependent und zwanghaft) genannt (Eher et al. 2010; Chantry und Craig 1994; McElroy et al. 1999; Raymond et al. 1999; Leue et al. 2004). In den bereits erwähnten österreichischen Studien von Eher et al. wurden bei $68 \%$ (Eher et al. 2010) bzw. 54\% (Eher et al. 2019) der untersuchten Sexualstraftäter Persönlichkeitsstörungen diagnostiziert.

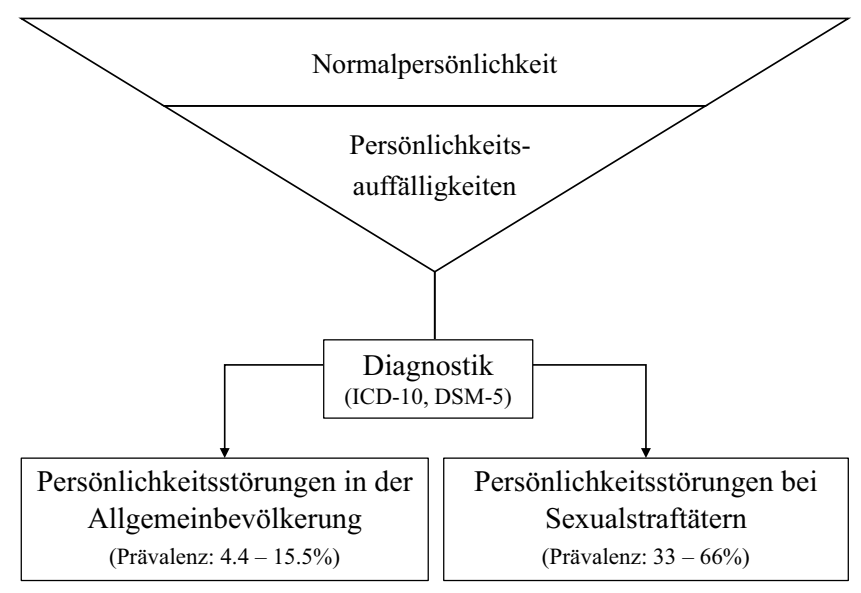

Abb. 2 Diagnostik und Zusammenhang zwischen Persönlichkeitsstörungen und Sexualdelinquenz

In Abb. 2 ist die Diagnostik von und der Zusammenhang zwischen Persönlichkeitsstörungen und Sexualdelinquenz dargestellt.

Auch hier gibt es bei bestimmten Delikten diagnostische Besonderheiten: Vergewaltiger weisen z.B. signifikant häufiger die Diagnose einer Persönlichkeitsstörung auf als Kindesmissbrauchstäter. Während bei Vergewaltigern Diagnosen aus dem Cluster B überrepräsentiert waren, fanden sich bei der letztgenannten Tätergruppe überproportional häufig Störungen aus dem Cluster C. Fand das Sexualdelikt innerhalb einer Intimpartnerschaft statt, erfüllten zwei Drittel der Täter die Kriterien einer Borderline-Persönlichkeitsstörung, während bei Tätern mit nichtfamiliären Opfern häufiger eine antisoziale Persönlichkeitsstörung vorlag (Eher et al. 2010, 2019). Die zwanghafte Persönlichkeitsstörung scheint eher bei Sexualstraftätern mit kindlichen als mit erwachsenen Opfern anzutreffen zu sein (Ahlmeyer et al. 2003; Eher et al. 2003; Prentky und Knight 1991).

Bei Sexualstraftätern, die ihre Opfer töten, wurde mit Raten zwischen 52 und $83 \%$ eine deutlich höhere Prävalenz an Persönlichkeitsstörungen beschrieben als bei Sexualstraftätern, die ihre Opfer nicht töten (4-58\%). Dabei waren antisoziale Persönlichkeitsstörungen bei Sexualmördern signifikant häufiger anzutreffen als bei Sexualstraftätern, die ihre Opfer nicht töten (Firestone et al. 1998a, 1998b; Koch et al. 2011; Langevin et al. 1988; Oliver et al. 2007). Insbesondere Störungen aus den Clustern A und B sind in der Gruppe der Sexualstraftäter mit Tötungsdelikten überrepräsentiert (Chan et al. 2015; Chan und Heide 2009; Hill et al. 2006; Chan und Beauregard 2016). Bei einem Vergleich sexuell sadistischer und nichtsadistischer Sexualmörder konnte gezeigt werden, dass Erstere häufiger eine antisoziale, schizotypische oder Borderline-Persönlichkeitsstörung aufwiesen (Hill et al. 2006). Gesamthaft scheinen antisoziale Persönlichkeitsstörungen, insbesondere die „psychopathy“ nach Hare (2003), die Wahrscheinlichkeit eines einschlägi- 
gen deliktischen Rückfalls zu erhöhen (Hanson und Morton-Bourgon 2005).

\section{Komorbidität von Persönlichkeits- und paraphilen Störungen bei Sexualdelinquenten}

Viele wissenschaftliche Untersuchungen ziehen Verbindungen zwischen Sexualstraftaten und bei den Tätern komorbid bestehenden Persönlichkeits- und paraphilen Störungen (z.B. Bogaerts et al. 2008; Leue et al. 2004; Ahlmeyer et al. 2003). Bogaerts et al. verglichen 36 paraphile mit 34 nichtparaphile Kindesmissbrauchstäter. Die als paraphil diagnostizierten Sexualstraftäter wiesen signifikant häufiger Persönlichkeitsstörungen auf als die nichtparaphilen. Komorbid traten v. a. Borderline-, histrionische, zwanghafte und depressive Persönlichkeitsstörungen auf (Bogaerts et al. 2008). In einer Untersuchung mit Sexualstraftätern aus dem deutschen Maßregelvollzug wurden bei paraphilen Kindesmissbrauchstätern im Vergleich zu denjenigen mit einer Störung der Impulskontrolle mit $74 \%$ vs. $40 \%$ knapp doppelt so häufig Persönlichkeitsstörungen diagnostiziert (Leue et al. 2004).

Es scheint darüber hinaus eine Verbindung zwischen komorbid auftretenden Persönlichkeits- und paraphilen Störungen und dem Rückfallrisiko zu geben. Bei der Psychopathy, welche als Unterform der antisozialen Persönlichkeitsstörung gilt, konnte eine Metaanalyse mit einer Gesamteffektgröße von $d=0,4$ ein schwachen, aber signifikanten Zusammenhang zwischen hohen Psychopathy-Werten und erhöhten Rückfallraten bei Sexualdelikten nachweisen. Dieser Effekt wurde besonders deutlich, wenn zudem paraphile Störungen sowie ein hoher Punktwert im Bereich antisozialer Lebensstil vorlagen (Hawes et al. 2013). Dieser Befund deckt sich mit den Erkenntnissen einer weiteren Metaanalyse, die ebenfalls normabweichende sexuelle Präferenzen und eine antisoziale Grundhaltung als wichtigste Prädiktoren für Rückfälle mit Sexualdelikte identifizierten (Hanson und Morton-Bourgon 2005). Diesem Befund wird auch in aktuarischen ,risk-assessment“-Instrumenten Rechnung getragen: So erhöhen im Sex Offender Risk Appraisal Guide (SORAG; Quinsey et al. 2006) die Diagnose einer Persönlichkeitsstörung, ein hoher Psychopathy-Wert und die Diagnose einer paraphilen Störung den Punktewert und damit die Risikokategorie.

\section{Implikationen für die therapeutische Praxis}

Moderne Straftäterbehandlung orientiert sich am „Riskneed-responsivity“-Prinzip, das die Ausrichtung von Maßnahmen am Rückfallrisiko der TäterInnen (Risk), an den zur Delinquenz beitragenden Faktoren (Need) sowie an der Passung zwischen Interventionen und Täterprofil (Responsivity) betont (Bonta und Andrews 2007).

Unter Berücksichtigung der referierten Forschungsergebnisse kann festgehalten werden, dass bei vielen Sexualstraftätern unterschiedliche Persönlichkeits- und paraphile Störungen zu finden sind. Diese stehen wiederum in Verbindung mit affektiven Symptomen, impulsivem und zwanghaftem Verhalten (Briken und Kafka 2007; Hoyer et al. 1999, 2001). Neben der Störungsebene spielen im Rahmen der Fallkonzeption Aspekte wie Selbstkontrolle, Bindungsfähigkeit oder soziale Kompetenz eine Rolle (Berner 2011; Briken 2017). Diese beiden Bereiche beeinflussen sich wechselseitig und sollten bei der Behandlungsplanung stets mitgedacht werden. Das Zusammenspiel von Persönlichkeits- und paraphilen Störungen sowie den dazugehörigen psychosozialen Funktionsbereichen im Rahmen der Behandlungskonzeption wird aus Abb. 3 ersichtlich.

So reagieren Menschen mit Cluster-C-Persönlichkeitsstörungen auf Konflikte mit Selbstzweifel und sind in ihren Beziehungskompetenzen mitunter eingeschränkt. Aufgrund ihres prinzipiell vorhandenen Beziehungswunsches und ihrer eher hohen Selbstkontrolle haben sie jedoch tendenziell ein niedriges Risiko für gewalttätige Sexualdelinquenz. Dahingegen legen Personen mit dissozialen, schizoiden und paranoiden Persönlichkeitsstörungen oftmals sowohl in Hinsicht auf ihren Selbstwert als auch im Rahmen zwischenmenschlicher Beziehungen einen malignen Narzissmus an den Tag, der bei parallelem Vorliegen psychopathischer Persönlichkeitsmerkmale (Hawes et al. 2013) und als ich-synton erlebten paraphilen Störungen ein besonders hohes Risiko für Sexualdelikte bedingt (Briken 2017). Gerade bei paraphilen Menschen mit antisozialer Persönlichkeitsstörung kann die mit der Persönlichkeitsstörung verbundene Impulsivität enthemmend wirken und so zum Tatgeschehen beitragen (Briken et al. 2010; Kröber 1997; Leygraf 2006; Hoyer 2001).

Tatsächlich stehen bei der Mehrzahl der Sexualstraftaten nicht zwingend sexuelle Motive im Vordergrund, stattdessen sind insbesondere bei Sexualdelikten an Erwachsenen häufig Dominanzstreben oder Aggressionen bedeutsamer (Füllgrabe 1983). So kann sich eine Person mit einer dissozialen Persönlichkeitsstörung immer wieder in unterschiedliche kriminelle Aktivitäten verstricken und ihre emotionsarme Bedürfnisorientierung oder Impulsivität auch im sexuellen Bereich ausleben, etwa in Form von Vergewaltigungen. Dies könnte auch einen Einfluss darauf haben, dass Personen mit antisozialen Persönlichkeitseigenschaften eher Personen außerhalb des familiären Rahmens schädigen (Seto et al. 2015).

Des Weiteren können fehlende soziale Kompetenzen und mangelnde Fähigkeiten der Emotionsregulation (Lussier et al. 2001) im Rahmen von Persönlichkeitsstörungen den 
Abb. 3 Zusammenspiel von Persönlichkeits- und paraphilen Störungen und psychosozialen Funktionsbereichen in der Behandlungskonzeption
Fall-/Behandlungskonzeption

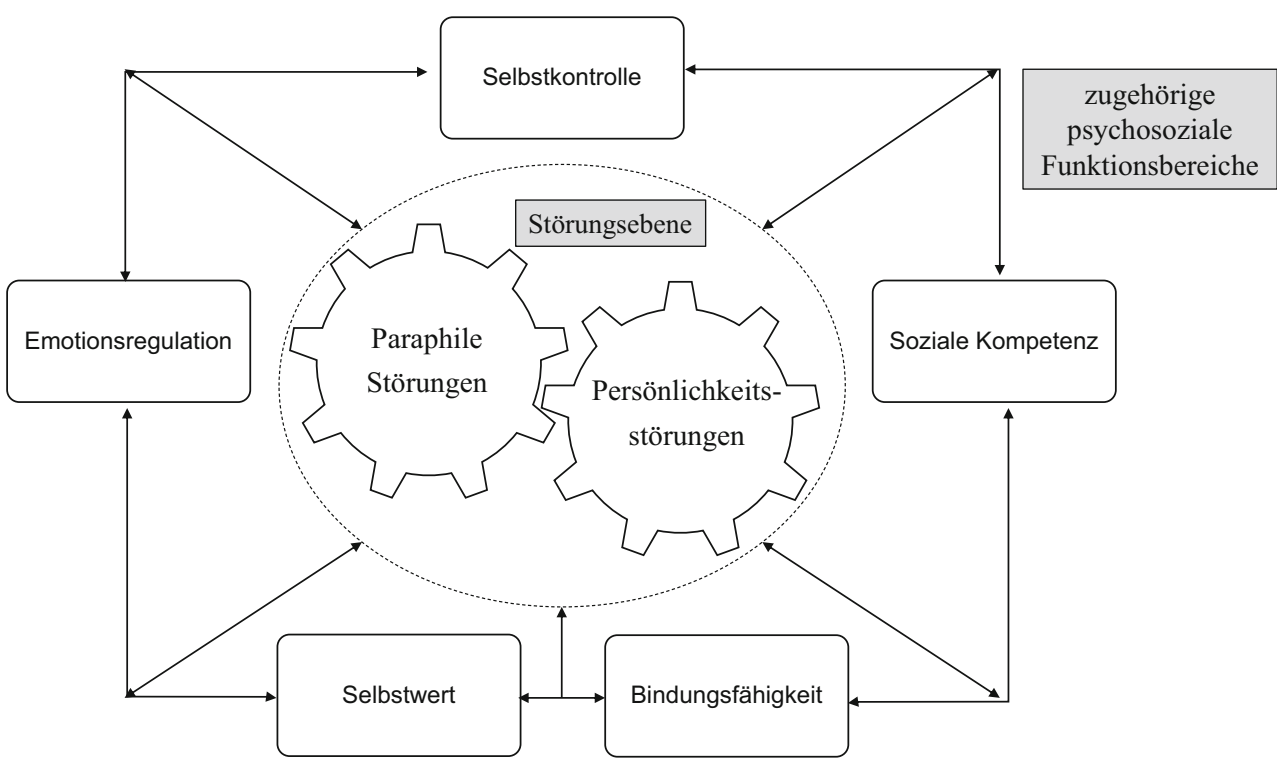

Zusammenhang zwischen psychischen Störungen und Sexualdelinquenz moderieren. Darunter fallen beispielsweise ein Mangel an Intimität und Sexualität, unbefriedigende zwischenmenschliche Beziehungen oder auch soziale Isolation (Marshall 1989). So treten Kindesmissbrauchstäter im Vergleich zu Vergewaltigern eher selbstunsicher, affektlabil, in sozialen Belangen ängstlich-vermeidend auf und sind sexuell unerfahren. Eventuell weichen sozial unbeholfene, gehemmt-selbstunsichere Kindesmissbrauchstäter infolge ihres Scheiterns in Beziehungen mit erwachsenen Personen auf Kinder aus, da diese als zugänglicher, manipulierbarer und weniger zurückweisend erlebt werden (Hoyndorf et al. 1995; Fiedler 2004; Marshall und Marshall 2000). Diese Täter tendieren dazu, Kinder aus dem familiären Umfeld zu schädigen. Das Risiko ist erhöht, wenn zudem soziale und familiäre Probleme bestehen (Seto et al. 2015).

Je nach Zusammenspiel der Persönlichkeits- und paraphilen Störungen und der Deliktdynamik sind die Behandlungsziele unterschiedlich auszurichten: Verbesserung der Selbstregulation und Umgang mit innerer Anspannung bei Borderline-Persönlichkeitsstörungen, Erhöhung der Beziehungs- und Bindungsfähigkeit bei schizoiden Personen, ein verbesserter Umgang mit Sexualität durch Psychoedukation bei unreifen Persönlichkeiten, Selbstreflexion und eine dialektische Betrachtung in der Balance zwischen Akzeptanz und Veränderung bei Pädophilie. Von allgemeiner Relevanz sind die Etablierung von Handlungsalternativen, Nutzung von Ressourcen und Entwicklung von Lebenszielen im Sinne des ,good lives model“ (Ward und Brown 2004; Ward et al. 2007).

\section{Limitationen bestehender Studien}

Studien, die sich mit den Zusammenhängen von paraphilen und Persönlichkeitsstörungen mit strafrechtlich relevantem Sexualverhalten befassen, verwenden in der Regel Stichproben, die sich aus verurteilten Straftätern zusammensetzen. Der größte Teil der vorliegenden Daten bezieht sich also auf das kriminologische Hellfeld. Forschende weisen außerdem immer wieder auf die vielfältigen methodischen Schwierigkeiten hin, mit denen sich Studien zu den $\mathrm{Zu}$ sammenhängen zwischen psychischen Störungen und Sexualdelinquenz konfrontiert sehen (Eher et al. 2019; Okami und Goldberg 1992; Krueger und Kaplan 2001): Diese betreffen kleine, vorselektierte Stichproben mit meist ausschließlich männlichen Probanden, unstrukturiertes Assessment psychischer Störungen, fehlende Untergruppenanalysen und aktenbasierte Vorgehensweisen. Oftmals wurden in den Studien keine Kontrollgruppen verwendet und weitere beeinflussende Faktoren wie situative Prädiktoren nicht berücksichtigt (Chan und Beauregard 2016). Bei Interviews bleibt gelegentlich eine Überprüfung der Beurteilungsübereinstimmung aus (Eher et al. 2010, 2019). Auch die retrospektiven und oftmals als Querschnitt angelegten Studiendesigns stellen ein Problem dar, da sie nur begrenzt kausale und funktionale Analysen zulassen (Briken et al. 2010; Fiedler 2004).

Einigen der vorgenannten Kritikpunkte begegneten Eher et al. mit einem Untersuchungsdesign, das repräsentative Daten österreichischer verurteilter Sexualstraftäter anstelle einer hochselektierten psychiatrischen Stichprobe verwendet (Eher et al. 2010, 2019). Diagnostisch können sich die beiden Studien auf die akten- und explorationsbasierte, kon- 
sensual erzielte Diagnostik erfahrener ForensikerInnen stützen, wodurch im Vergleich zu reinen Aktenerhebungen eine hohe Aussagekraft der Daten gewährleistet ist.

\section{Fazit}

Unter Sexualstraftätern konnte eine im Vergleich zur Allgemeinbevölkerung erhöhte Prävalenz psychischer Störungen festgestellt werden, darunter v.a. Persönlichkeits- und paraphile Störungen. Sexualstraftäter mit paraphilen Störungen, vorwiegend Kindesmissbrauchstäter und Sexualmörder, zeigen vermehrt psychosoziale Auffälligkeiten und psychiatrische Komorbiditäten. Die häufigste paraphile Störung bei Sexualstraftätern mit Kindern als Opfer ist erwartungsgemäß die Pädophilie. Für Täter mit erwachsenen Opfern spielt v. a. der sexuelle Sadismus eine Rolle. Auch die Prävalenz der Persönlichkeitsstörungen ist unter Sexualstraftätern im Vergleich zur Allgemeinpopulation erhöht. Dies gilt unabhängig vom Delikttyp insbesondere für die antisoziale Persönlichkeitsstörung. Während Vergewaltiger und Sexualmörder häufiger Persönlichkeitsstörungen und vorwiegend solche aus dem Cluster B bzw. paranoide und schizotypische zeigen, betreffen die Persönlichkeitsstörungen von Kindesmissbrauchstätern häufig das Cluster $\mathrm{C}$ mit insbesondere dependenten und zwanghaften Ausprägungen.

Derzeit kann nicht eindeutig geschlussfolgert werden, wie genau psychische Störungen und sexuell delinquentes Verhalten zusammenhängen. Schließlich können auch situativ-kontextuelle Bedingungen tatbegünstigend wirken (Fiedler 2004). Entsprechend bieten Persönlichkeits- und paraphile Störungen in der Regel keine monokausale Erklärung für Sexualdelinquenz. Die sorgfältige Diagnostik entsprechender Auffälligkeiten stellt jedoch ein unverzichtbares Element für Risikoeinschätzung, Behandlung und Prognose von sexuell delinquentem Verhalten dar und bedarf daher besonderer Aufmerksamkeit (Moulden und Marshall 2017).

Funding Open Access funding provided by Projekt DEAL.

Interessenkonflikt T.A. Hörburger und E. Habermeyer geben an, dass kein Interessenkonflikt besteht.

Open Access Dieser Artikel wird unter der Creative Commons Namensnennung 4.0 International Lizenz veröffentlicht, welche die Nutzung, Vervielfältigung, Bearbeitung, Verbreitung und Wiedergabe in jeglichem Medium und Format erlaubt, sofern Sie den/die ursprünglichen Autor(en) und die Quelle ordnungsgemäß nennen, einen Link zur Creative Commons Lizenz beifügen und angeben, ob Änderungen vorgenommen wurden.

Die in diesem Artikel enthaltenen Bilder und sonstiges Drittmaterial unterliegen ebenfalls der genannten Creative Commons Lizenz, sofern sich aus der Abbildungslegende nichts anderes ergibt. Sofern das betreffende Material nicht unter der genannten Creative Commons Lizenz steht und die betreffende Handlung nicht nach gesetzlichen Vorschrif- ten erlaubt ist, ist für die oben aufgeführten Weiterverwendungen des Materials die Einwilligung des jeweiligen Rechteinhabers einzuholen.

Weitere Details zur Lizenz entnehmen Sie bitte der Lizenzinformation auf http://creativecommons.org/licenses/by/4.0/deed.de.

\section{Literatur}

Abel GG, Mittelman M, Becker JV (1985) Sex offenders: Results of assessment and recommendations for treatment. In: Ben-Aron $\mathrm{MH}$ Hucker SJ, Webster CD (Hrsg) Clinical criminology: The assessment and treatment of criminal behavior. M\&M Graphics, Toronto, ON, Canada, S 207-220

Agnew J (2001) An overview of paraphilia. Venereology 14(4):148

Ahlers CJ, Schaefer GA, Mundt IA, Roll S, Englert H, Willich SN, Beier KM (2011) How unusual are the contents of paraphilias? Paraphilia-associated sexual arousal patterns in a community-based sample of men. J Sex Med 8(5):1362-1370

Ahlmeyer S, Kleinsasser D, Stoner J, Retzlaff P (2003) Psychopathology of incarcerated sex offenders. J Pers Disord 17(4):306-318

Al-Rousan T, Rubenstein L, Sieleni B, Deol H, Wallace RB (2017) Inside the nation's largest mental health institution: a prevalence study in a state prison system. BMC Public Health 17(1):342

American Psychiatric Association (2014) Diagnostisches und statistisches manual psychischer Störungen - DSM-5 ${ }^{\circledR}$. Hogrefe, Göttingen

Barnow S, Stopsack M, Ulrich I, Falz S, Dudeck M, Spitzer C, Grabe H-J, Freyberger HJ (2010) Prävalenz und Familiarität von Persönlichkeitsstörungen in Deutschland: Ergebnisse der Greifswalder Familienstudie. Psychother Psychosom Med Psychol 60(09/10):334-341

Berger P, Berner W, Bolterauer J, Gutierrez K, Berger K (1999) Sadistic personality disorder in sex offenders: Relationship to antisocial personality disorder and sexual sadism. J Personal Disord 13(2):175-186

Berner W (2011) Perversion. Psychosozial-Verlag, Gießen

Berner W, Berger P, Guitierrez K, Jordan B, Berger K (1992) The role of personality disorders in the treatment of sex offenders. J Offender Rehabil 18(3-4):25-37

Berner W, Berger P, Hill A (2003) Sexual sadism. Int J Offender Ther Comp Criminol 47(4):383-395

Bogaerts S, Daalder A, Vanheule S, Desmet M, Leeuw F (2008) Personality disorders in a sample of paraphilic and nonparaphilic child molesters: a comparative study. Int J Offender Ther Comp Criminol 52(1):21-30

Bonta J, Andrews DA (2007) Risk-need-responsivity model for offender assessment and rehabilitation. Rehabilitation 6(1):1-22

Bradford JM, Boulet J, Pawlak A (1992) The paraphilias: a multiplicity of deviant behaviours. Can J Psychiatry 37(2):104-108

Briken P (2017) Antisoziale Persönlichkeitsstörung und Sexualität. In: Dulz B, Briken P, Kernberg OF, Rauchfleisch U (Hrsg) Handbuch der Antisozialen Persönlichkeitsstörung. Schattauer, Stuttgart, S 369-380

Briken P, Kafka MP (2007) Pharmacological treatments for paraphilic patients and sexual offenders. Curr Opin Psychiatry 20(6):609-613

Briken P, Habermann N, Kafka MP, Berner W, Hill A (2006) The paraphilia-related disorders: an investigation of the relevance of the concept in sexual murderers. J Forensic Sci 51(3):683-688

Briken P, Hill A, Habermann N, Kafka MP, Berner W (2010) Paraphilia-related disorders and personality disorders in sexual homicide perpetrators. Sex Offender Treat 5(1):1-7

Chan HC, Beauregard E (2016) Non-homicidal and homicidal sexual offenders: prevalence of maladaptive personality traits and paraphilic behaviors. J Interpers Violence 31(13):2259-2290

Chan HC, Heide KM (2009) Sexual homicide: a synthesis of the literature. Trauma Violence Abuse 10(1):31-54 
Chan HC, Beauregard E, Myers WC (2015) Single-victim and serial sexual homicide offenders: differences in crime, paraphilias and personality traits. Crim Behav Ment Health 25(1):66-78

Chantry K, Craig RJ (1994) Psychological screening of sexually violent offenders with the MCMI. J Clin Psychol 50(3):430-435

Dawson SJ, Bannerman BA, Lalumière ML (2016) Paraphilic interests: an examination of sex differences in a nonclinical sample. Sex Abuse 28(1):20-45

Dilling H, Freyberger HJ (2012) Taschenführer zur ICD-10-Klassifikation psychischer Störungen. Huber, Bern

Dombert B, Schmidt AF, Banse R, Briken P, Hoyer J, Neutze J, Osterheider M (2016) How common is men's self-reported sexual interest in prepubescent children? J Sex Res 53(2):214-223

Dunsieth NW, Nelson EB, Brusman-Lovins LA, Holcomb JL, Beckman D, Welge JA, Roby D, Taylor P Jr, Soutullo CA, McElroy SL (2004) Psychiatric and legal features of 113 men convicted of sexual offenses. J Clin Psychiatry 65(3):293

Eher R, Neuwirth W, Fruehwald S, Frottier P (2003) Sexualization and lifestyle impulsivity: clinically valid discriminators in sexual offenders. Int J Offender Ther Comp Criminol 47(4):452-467

Eher R, Rettenberger M, Schilling F (2010) Psychiatrische Diagnosen von Sexualstraftätern. Z Sex-Forsch 23(01):23-35

Eher R, Rettenberger M, Turner D (2019) The prevalence of mental disorders in incarcerated contact sexual offenders. Acta Psychiatr Scand 193(6):572

Fazel S, Danesh J (2002) Serious mental disorder in 23000 prisoners: a systematic review of 62 surveys. Lancet 359(9306):545-550

Fazel S, Sjöstedt G, Långström N, Grann M (2007) Severe mental illness and risk of sexual offending in men: a case-control study based on Swedish national registers. J Clin Psychiatry 68(4):588

Fiedler P (2004) Die Bedeutung psychischer Störungen für Sexualdelinquenz

Firestone P, Bradford JM, Greenberg DM, Larose MR (1998a) Homicidal sex offenders: psychological, phallometric, and diagnostic features. J Am Acad Psychiatry Law 26(4):537-552

Firestone P, Bradford JM, Greenberg DM, Larose MR, Curry S (1998b) Homicidal and nonhomicidal child molesters: psychological, phallometric, and criminal features. Sex Abuse 10(4):305-323

Fromberger P, Jordan K, Müller J (2013) Pädophilie. Nervenarzt 84(9):1123-1135

Füllgrabe U (1983) Kriminalpsychologie. Verlag für Angewandte Psychologie, Göttingen

Hanson RK, Morton-Bourgon KE (2005) The characteristics of persistent sexual offenders: a meta-analysis of recidivism studies. J Consult Clin Psychol 73(6):1154-1163. https://doi.org/10.1037/ 0022-006X.73.6.1154

Hare RD (2003) Manual for the revised psychopathy checklist, 2. Aufl. Multi-Health Systems, Toronto

Hawes SW, Boccaccini MT, Murrie DC (2013) Psychopathy and the combination of psychopathy and sexual deviance as predictors of sexual recidivism: meta-analytic findings using the Psychopathy Checklist-Revised. Psychol Assess 25(1):233-243. https://doi. org/10.1037/a0030391

Helmus L, Hanson RK (2009) Thornton D Reporting Static-99 in light of new research on recidivism norms. In: The forum, Bd. 1, S $38-45$

Hill A, Habermann N, Berner W, Briken P (2006) Sexual sadism and sadistic personality disorder in sexual homicide. J Personal Disord 20(6):671-684

Hoyer J (2001) Psychodiagnostische Kategorisierung von gefährlichen Sexualdelinquenten. Psychische Störungen bei Sexualdelinquenten. Pabst, Lengerich, S 13-31

Hoyer J, Kunst H, Borchard B, Stangier U (1999) Paraphile versus impulskontrollgestörte Sexualstraftäter: Eine psychologisch valide Differenzierung? Z Klin Psychol 28(1):37-44

Hoyer J, Kunst H, Schmidt A (2001) Social phobia as a comorbid condition in sex offenders with paraphilia or impulse control disorder. J Nerv Ment Dis 189(7):463-470
Hoyndorf S, Reinhold M, Christmann F (1995) Behandlung sexueller Störungen. Beltz/PVU, Weinheim

Jackson RL, Richards HJ (2007) Diagnostic and risk profiles among civilly committed sex offenders in Washington State. Int J Offender Ther Comp Criminol 51(3):313-323

Khachatryan N, Heide KM, Hummel EV, Chan HC (2016) Juvenile sexual homicide offenders: thirty-year follow-up investigation. Int J Offender Ther Comp Criminol 60(3):247-264

Koch J, Berner W, Hill A, Briken P (2011) Sociodemographic and diagnostic characteristics of homicidal and nonhomicidal sexual offenders. J Forensic Sci 56(6):1626-1631

Kröber H (1997) Strafrechtliche Begutachtung von Persönlichkeitsstörungen. Persönlichkeitsstörungen Theor Ther 1:161-171

Krueger RB, Kaplan MS (2001) The paraphilic and hypersexual disorders: an overview. J Psychiatr Pract 7(6):391-403

Laajasalo T, Ylipekka M, Häkkänen-Nyholm H (2013) Homicidal behaviour among people with avoidant, dependent and obsessive-compulsive (cluster C) personality disorder. Crim Behav Ment Health 23(1):18-29

Langevin R, Ben-Aron M, Wright P, Marchese V, Handy L (1988) The sex killer. Ann Sex Res 1(2):263-301

Leue A, Borchard B, Hoyer J (2004) Mental disorders in a forensic sample of sexual offenders. Eur Psychiatry 19(3):123-130

Leygraf N (2006) Psychisch kranke Rechtsbrecher. In: Handbuch der Forensischen Psychiatrie. Springer, Berlin Heidelberg, S 254-270

Lussier P, Proulx J, McKibben A (2001) Personality characteristics and adaptive strategies to cope with negative emotional states and deviant sexual fantasies in sexual aggressors. Int $\mathrm{J}$ Offender Ther Comp Criminol 45(2):159-170

Långström N, Sjöstedt G, Grann M (2004) Psychiatric disorders and recidivism in sexual offenders. Sex Abuse 16(2):139-150

Marshall WL (1989) Intimacy, loneliness and sexual offenders. Behaviour Research and Therapy 27 (5):491-504

Marshall WL, Marshall LE (2000) The origins of sexual offending. Trauma Violence Abuse 1(3):250-263

McElroy SL, Soutullo CA, Taylor P Jr, Nelson EB, Beckman DA, Brusman LA, Ombaba JM, Strakowski SM, Keck PE Jr (1999) Psychiatric features of 36 men convicted of sexual offenses. J Clin Psychiatry 60(6):414

Moulden HM, Marshall LE (2017) Major mental illness in those who sexually abuse. Curr Psychiatry Rep 19(12):105

Myers WC, Chan HC, Mariano TY (2016) Sexual homicide in the USA committed by juveniles and adults, 1976-2007: age of arrest and incidence trends over 32 years. Crim Behav Ment Health 26(1):38-49

Myers WC, Chan HC, Vo EJ, Lazarou E (2010) Sexual sadism, psychopathy, and recidivism in juvenile sexual murderers. J Investig Psychol Offender Profiling 7(1):49-58

Niemeczek A (2015) Phänomen der Sexualdelinquenz. In: Tatverhalten und Täterpersönlichkeit von Sexualdelinquenten. Springer, Berlin Heidelberg, S 17-39

Okami P, Goldberg A (1992) Personality correlates of pedophilia: are they reliable indicators? J Sex Res 29(3):297-328

Oliver CJ, Beech AR, Fisher D, Beckett R (2007) A comparison of rapists and sexual murderers on demographic and selected psychometric measures. Int $\mathrm{J}$ Offender Ther Comp Criminol 51(3):298-312

Osterheider M, Banse R, Briken P, Goldbeck L, Hoyer J, Santtila P, Eisenbarth H (2012) Häufigkeit, Erklärungsmodelle und Folgen sexueller Gewalt an Kindern und Jugendlichen: Zielsetzungen des deutschlandweiten MiKADO-Projekts. Z Sex-Forsch 25(03):286-292

Prentky RA, Knight RA (1991) Identifying critical dimensions for discriminating among rapists. J Consult Clin Psychol 59(5):643

Quinsey VL, Harris GT, Rice ME, Cormier CA (2006) Violent offenders: appraising and managing risk. American Psychological Association, Washington DC 
Raymond NC, Coleman E, Ohlerking F, Christenson GA, Miner M (1999) Psychiatric comorbidity in pedophilic sex offenders. Am J Psychiatry 156(5):786-788

Seto MC, Babchishin KM, Pullman LE, McPhail IV (2015) The puzzle of intrafamilial child sexual abuse: a meta-analysis comparing intrafamilial and extrafamilial offenders with child victims. Clin Psychol Rev 39:42-57

Steel Z, Marnane C, Iranpour C, Chey T, Jackson JW, Patel V, Silove D (2014) The global prevalence of common mental disorders: a systematic review and meta-analysis 1980-2013. Int J Epidemiol 43(2):476-493

Ward T, Brown M (2004) The good lives model and conceptual issues in offender rehabilitation. Psychol Crime Law 10(3):243-257
Ward T, Mann RE, Gannon TA (2007) The good lives model of offender rehabilitation: clinical implications. Aggress Violent Behav 12(1):87-107

Watts AL, Nagel MG, Latzman RD, Lilienfeld SO (2019) Personality disorder features and paraphilic interests among undergraduates: differential relations and potential antecedents. J Personal Disord 33(1):22-48

Williams KM, Cooper BS, Howell TM, Yuille JC, Paulhus DL (2009) Inferring sexually deviant behavior from corresponding fantasies: the role of personality and pornography consumption. Crim Justice Behav 36(2):198-222 